\title{
Optimal Energy Management of Two Stage Energy Distribution Systems Using Clustering Algorithm
}

\author{
A. Y. S. Pandiyan ${ }^{1}$, M. E. Kiziroglou ${ }^{1}$, D. E. Boyle ${ }^{2}$, S.W. Wright ${ }^{1}$ and E. M. Yeatman \\ ${ }^{1}$ Department of Electronic and Electrical Engineering, Imperial College London, UK. \\ ${ }^{2}$ Dyson School of Design Engineering, Imperial College London, UK
}

\begin{abstract}
Motivated by recent developments in Wireless Power Transfer (WPT), this work presents a solution for the optimization of a two-stage energy distribution system combining inductive and acoustic power transfer using a clustering algorithm. A network of immobile wireless sensors equipped with acoustic transceivers, storage capacitors and with known cartesian coordinates in a 2D plane is considered. A power delivery vehicle (PDV) with finite energy storage capacity is used to recharge a sensor node's supercapacitor which then transmits power to neighboring sensors acoustically within range. This work aims to find an optimal charging route for the PDV. The proposed algorithm is a combination of cluster analysis and breadth-first search. A theoretical study was performed, and the simulation results obtained were studied for the long-term failure probability for the proposed energy scheme.
\end{abstract}

\section{Motivation}

Among the various WPT methods, inductive coupling has been widely investigated. There are already commercially available products such as phone chargers. However, the efficiency of inductive transfer decreases rapidly with increasing distance due to poor coupling between the coils. To overcome the mobility issue, use of power delivery vehicles (like drones) for powering remote network systems has also been of recent interest [1]. However, charging every node in a wireless sensor network (WSN) increases the travel time of the PDV and therefore heavily increases its energy consumption, requiring frequent drone charging. To address this problem, the authors in [2] proposed a novel 'Two-Stage Power Distribution' scheme that uses both inductive and acoustic coupling to transfer power wirelessly to remote nodes inaccessible to the PDV (Fig. 1). The main idea is that the PDV recharges selective/accessible nodes in the WSN, each of which in turns drives an acoustic transducer that transmits a part of this energy through sound waves using a common medium to the neighboring nodes. In this work, the methods to optimize the energy distribution in such a system are simulated and analyzed.

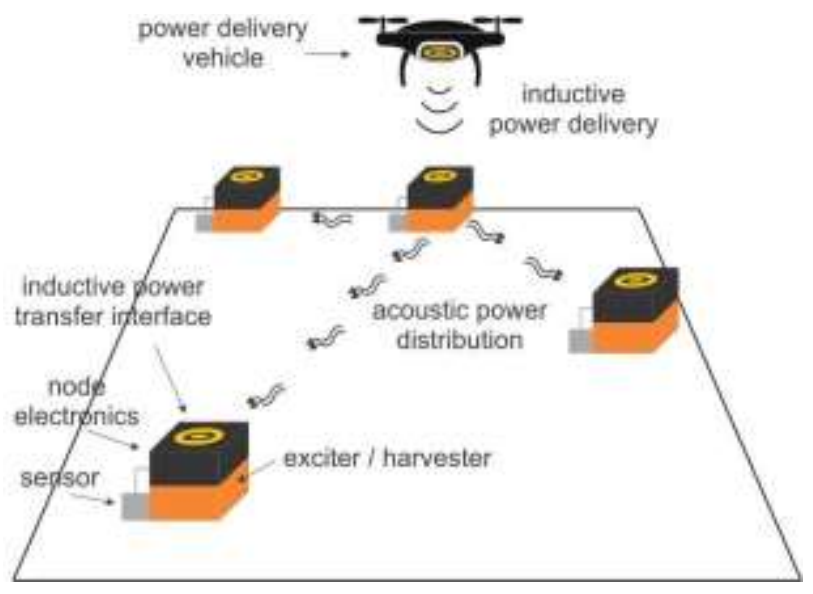

Fig. 1 Two Stage Power Delivery Scheme proposed in [2], using Inductive and Acoustic Power Transfer across a wireless network.

\section{System model}

Throughout this paper, a WSN comprising $N_{S}$ sensor nodes is assumed, with their known 2-D cartesian coordinates and initial storage energy. Both their position and initial energy levels are randomized to generate various scenarios during the simulations. It is also presumed that all the nodes of the WSN are equipped with similar components and system blocks. A block diagram of a node is shown in Fig. 2. During operation, a sensor node is considered either to be in sensing mode or power distribution mode. In sensing mode, the sensor utilizes energy $E_{m}$ from the storage capacitor to make measurements, process the data and communicate information to the base station. In the power distribution mode, the network cluster is expected to receive energy inductively (center node) and distribute power acoustically to the end nodes in the cluster. The center nodes receiving 'energy packets' $(I)$ from the PDV are always assumed to recharge the storage capacitor to their maximum energy. This ensures that there is enough power to drive the acoustic transmitter. 
The WSN is clustered based on the current energy level of the sensor node and the distance of the cluster center with respect to the others. The main idea of the cluster is to maximize the number of sensor nodes that benefit from the acoustic energy distribution [3]. For this, the radius of the cluster is set to the maximum distance that a minimum amount of acoustic energy can be transmitted. For a plane much larger than the acoustic limit, multiple clusters are generated to obtain a better control strategy. A factor of criticality is also imposed, where energizing a node by inductive transfer is prioritized when it fails to make a measurement/communicate for a long time. The energy of the storage capacitor $E_{S C}$ at any time $t$ for any node $n$, transmitting power $T$ to the other sensors, and receiving power $R$ acoustically from other sensors assuming a time increment of $1 \mathrm{sec}$, can be expressed as

$$
E_{S C}(t)=\min \left\{E_{S C}(t-1)-E_{m}-T+I+R, E_{S C_{M A X}}\right\}
$$

Iterative execution of these conditions would generate a set of clusters, whose center coordinates define the node best-placed to receive the energy packets from the PDV. This node will also be responsible for the acoustic distribution of energy. Implementation of a breadth-first search [4] to this array of cluster centers will generate an optimized path for the PDV. This leads to an optimal power control scheme where the PDV uses a clustering algorithm to minimize the acoustic and inductive channel losses and maximize the energy supplied to the sensor nodes in each PDV recharge cycle.

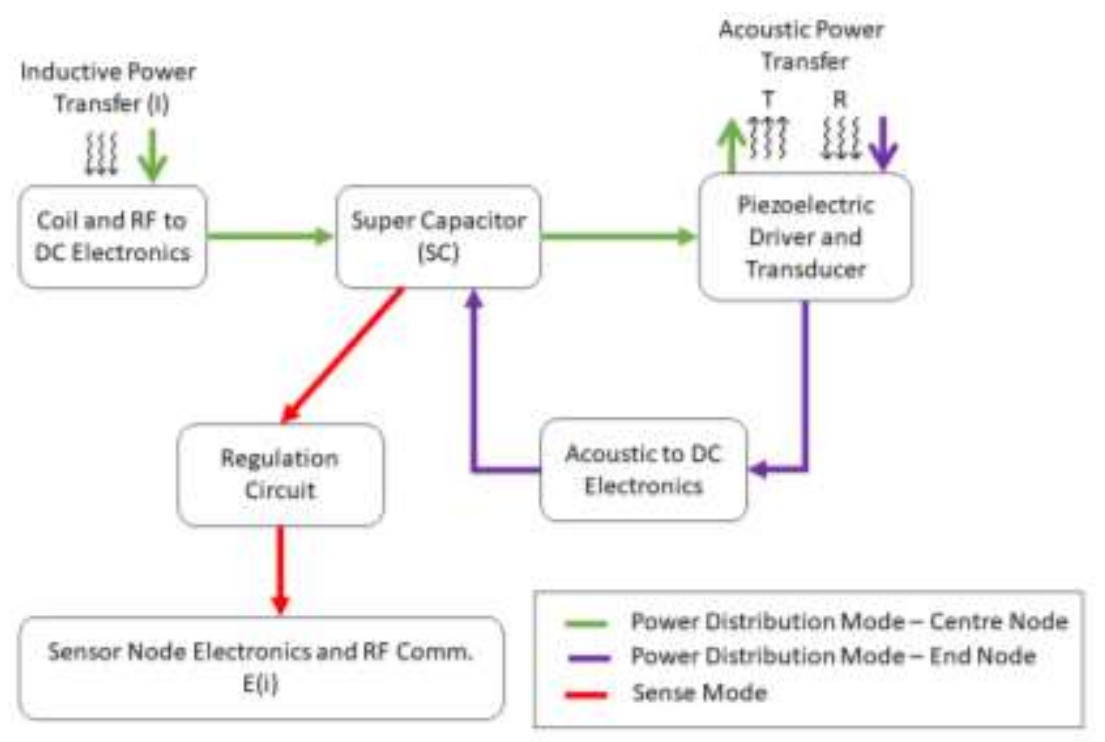

Fig. 2: Block diagram of a sensor node including the inductive and acoustic electronics, representing the energy flow during different modes of operation.

\section{Simulation Result}

The described algorithm was used for the simulation of a pressure sensor network, the specifications of which are summarized in Table 1.

\begin{tabular}{|c|c|c|c|}
\hline \multicolumn{4}{|c|}{ Table 1: Specifications of the WSN components. } \\
\hline \multicolumn{2}{|c|}{ Parameters } & $\begin{array}{c}\text { Pressure Sensor Node } \\
{[5]}\end{array}$ & $\begin{array}{c}\text { Storage Capacitor } \\
{[6]}\end{array}$ \\
\hline \multicolumn{2}{|l|}{ Model No. } & NPA300B02WG & BZ125A503ZAB \\
\hline \multirow{2}{*}{ Supply Voltage } & Typ. & $3.3 \mathrm{~V}$ & $5 \mathrm{~V}$ \\
\hline & Max & $5 \mathrm{~V}$ & $5.5 \mathrm{~V}$ \\
\hline \multicolumn{2}{|l|}{ Typical Current } & $1.2 \mathrm{~mA}$ & -- \\
\hline \multicolumn{2}{|c|}{ Response Time } & $0.5 \mathrm{~ms}$ & -- \\
\hline \multicolumn{2}{|c|}{ Sampling Frequency } & $0.1 \mathrm{~Hz}$ & -- \\
\hline \multicolumn{2}{|l|}{ Pon Time $_{\text {on }}$} & $2 \mathrm{~ms}$ & -- \\
\hline \multicolumn{2}{|l|}{ Capacitance } & -- & $1 \mathrm{~F}$ \\
\hline \multicolumn{2}{|c|}{ Leakage Current } & -- & $10 \mu \mathrm{A}$ \\
\hline \multicolumn{2}{|c|}{ Energy per sample, stored } & $12 \mathrm{uJ}$ & $12.5 \mathrm{~J}-$ \\
\hline
\end{tabular}


The operation of the algorithm is as shown in Fig. 3, which is visualized for a WSN of 100 randomly placed sensor nodes. The path and the positions of the PDV are traced along the dashed line and thick black outlines respectively. The positions of the sensor nodes are mapped along a 2D plane of dimensions $10 \mathrm{~m}$ by $10 \mathrm{~m}$, with their respective energy levels charted in a color map. The red, dash-dotted circles embody the nodes receiving power through acoustic transmission which is limited by the radius of the acoustic distribution. An approximate radius of $1 \mathrm{~m}$ is considered in the simulation corresponding to the experimental results from [7].

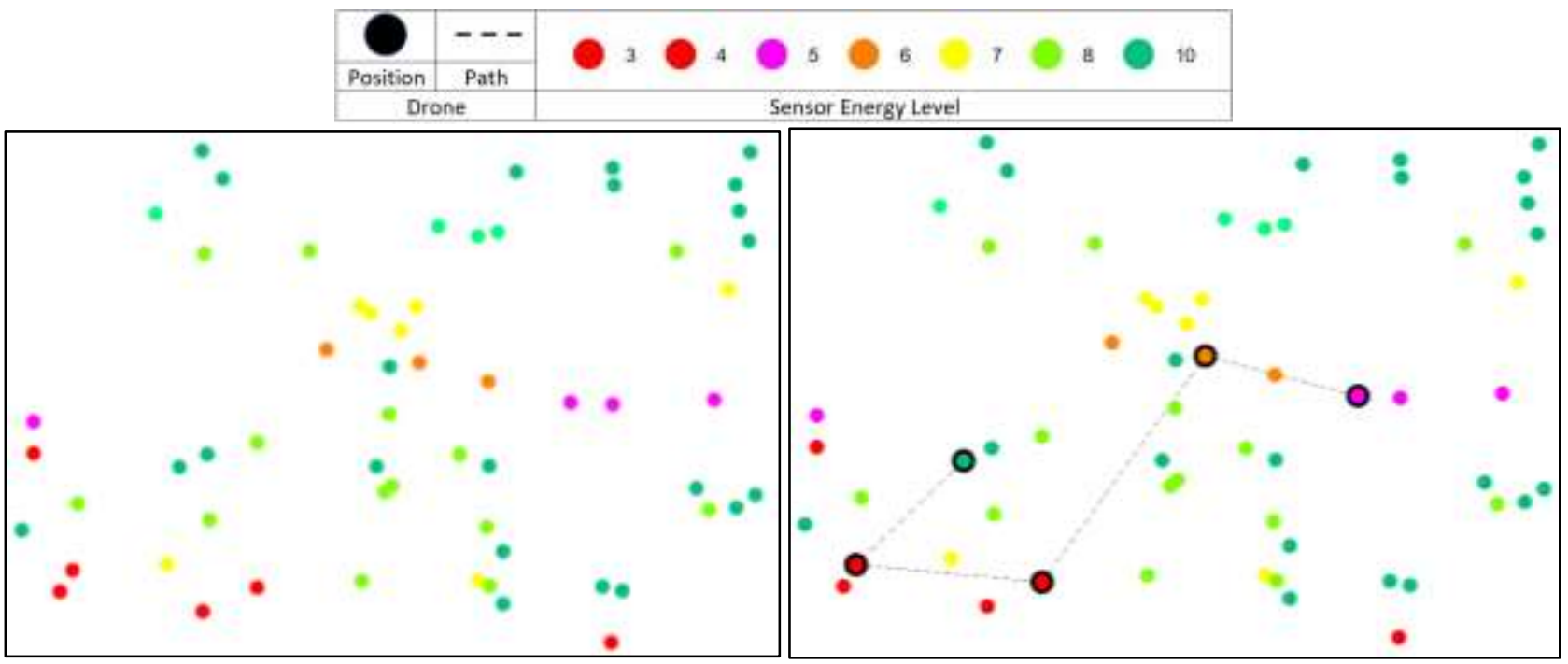

(a)

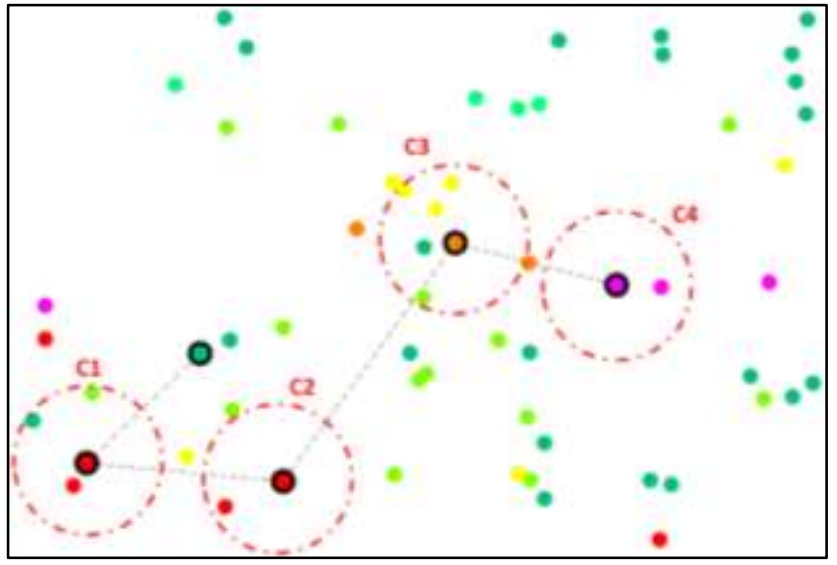

(c) (b)

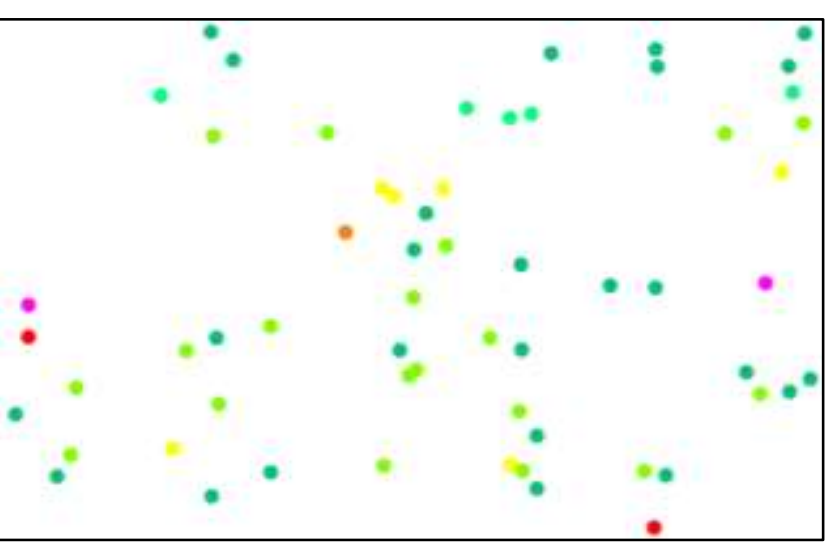

(d)

Fig. 3: Visualization of the stages of the two-stage power distribution scheme and the proposed control algorithm for a WSN in a plane dimension of $10 \mathrm{~m}$ by $10 \mathrm{~m}$. (a) Initial state of the system (b) The optimized drone path for Inductive power transfer (c) Clustering of the WSN for Acoustic power transfer. (d) The final state of the system.

It can be observed from the example visualized in Fig. 3(c) that the algorithm considers both these obligatory conditions efficiently: (a) to address critical nodes which can be seen from cluster 1 and 3; and (b) to choose an optimized center node in case of a dense cluster (C3). To test the efficiency of the proposed energy control scheme, the number of measurements missed by the sensor node is counted for two PDV cycles and the results are shown in Fig. 4. The term 'failure density' in this paper can be defined as the total number of times a sensor node fails to sense/transmit a measurement calculated over a one day period. The corresponding average storage capacitor energy levels of each sensor node in the network are also plotted for comparison. The high peak at the beginning of the graph is due to the randomized energy levels of the sensor nodes, and therefore initial stabilization was required. After this, the WSN appears to be autonomous until some nodes enter a critical state failing to make measurements, which in turn triggers a PDV recharging cycle. It is also noticeable that the discharge pattern of average energy in the WSN is identical between the two PDV visits. This is expected as the clustering is passive, resulting in a similar PDV recharge path during every cycle. Considering this as a pioneering attempt to develop an energy distribution strategy for WSN, adapting a dynamic clustering algorithm could be suggested as the next progressive step of this work. 

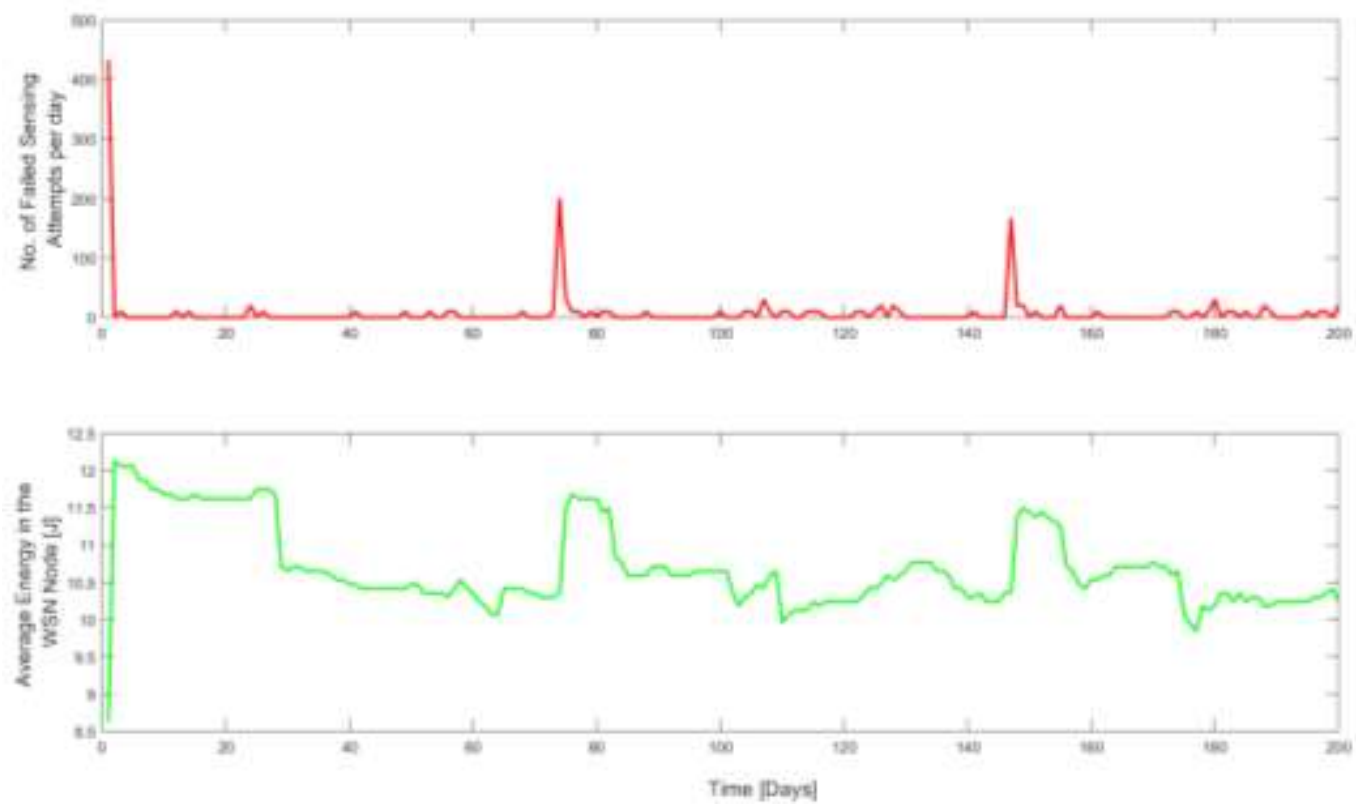

Fig. 4: Failure density of the WSN.

\section{Summary}

In this work, we considered a two-stage recharge scheme which uses both inductive and acoustic power transfer to wirelessly charge a wireless sensor network (WSN), thereby promoting their autonomous operation. An optimization algorithm for such a system to maximize the transferred power and minimize the wireless transfer losses is proposed. Physical conditions were set as control parameters for the algorithm to recognize critical conditions to trigger the recharge cycle. Through simulations, the working of the algorithm was demonstrated and its efficiency evaluated through failure analysis of the network. In this way, a new WPT energy solution for dense and conventionally inaccessible WSNs has been demonstrated. For future work, adapting dynamic control algorithms and studying the influence of various parameters (such as number of sensors, or density of WSN) would be beneficial in developing the control scheme.

\section{Acknowledgement}

The authors gratefully acknowledge the funding provided by the European Commission under the H2020 Marie Sklodowska Curie's ENHANCE project, Grant Agreement No: 722496 to accomplish this research.

\section{References}

[1] P. D. Mitcheson et al., "Energy-autonomous sensing systems using drones," in 2017 IEEE SENSORS, 2017, pp. 1-3.

[2] D. E. Boyle, S. W. Wright, M. E. Kiziroglou, A. Y. S. Pandiyan, and E. M. Yeatman, "Inductive Power Delivery with Acoustic Distribution to Wireless Sensors," in Wireless Power Week, London, UK, 2019.

[3] K. A. Darabkh, N. J. Al-Maaitah, I. F. Jafar, and A. F. Khalifeh, "Energy efficient clustering algorithm for wireless sensor networks," in 2017 International Conference on Wireless Communications, Signal Processing and Networking (WiSPNET), 2017, pp. 590-594.

[4] E. F. Moore, The Shortest Path Through a Maze. Bell Telephone System., 1959.

[5] Amphenol, Advanced, and Sensors. (11-Nov-2019). Available:

https://www.amphenol-sensors.com/en/novasensor/pressure-sensors/3161-npa-series

[6] Digikey. (10/11/2019). Available:

https://www.digikey.com/en/datasheets/avx-corporation/avx-corporation-bestcap

[7] M. E. Kiziroglou, D. E. Boyle, S. W. Wright, and E. M. Yeatman, "Acoustic energy transmission in cast iron pipelines," in Journal of Physics: Conference Series, 2015, vol. 660, no. 1, p. 012095: IOP Publishing.

Corresponding author: Akshayaa Y.S. Pandiyan, 707, Electrical Engineering, South Kensington, SW7 2BT London, UK. Phone: +44 (0) 207594 6242. Mail: a.pandiyan@imperial.ac.uk 